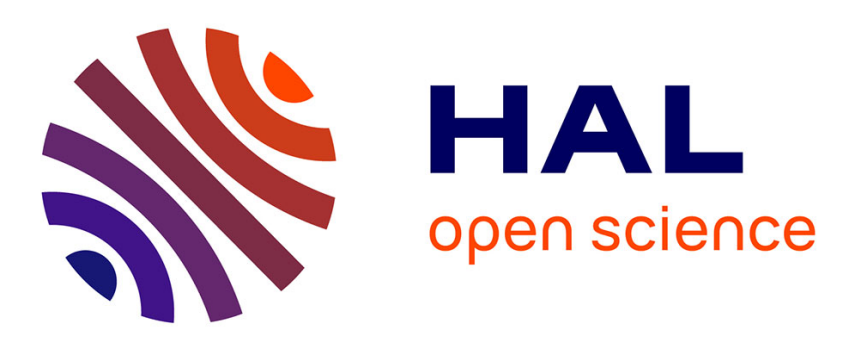

\title{
AMORPHOUS MAGNETIC FILMS FOR NON-STORAGE APPLICATIONS
}

\author{
H. Lachowicz, A. Milewski, J. Wenda
}

\section{To cite this version:}

H. Lachowicz, A. Milewski, J. Wenda. AMORPHOUS MAGNETIC FILMS FOR NONSTORAGE APPLICATIONS. Journal de Physique Colloques, 1985, 46 (C6), pp.C6-181-C6-188. 10.1051/jphyscol:1985631 . jpa-00224879

\section{HAL Id: jpa-00224879 https://hal.science/jpa-00224879}

Submitted on 1 Jan 1985

HAL is a multi-disciplinary open access archive for the deposit and dissemination of scientific research documents, whether they are published or not. The documents may come from teaching and research institutions in France or abroad, or from public or private research centers.
L'archive ouverte pluridisciplinaire HAL, est destinée au dépôt et à la diffusion de documents scientifiques de niveau recherche, publiés ou non, émanant des établissements d'enseignement et de recherche français ou étrangers, des laboratoires publics ou privés. 


\title{
AMORPHOUS MAGNETIC FILMS FOR NON-STORAGE APPLICATIONS
}

\author{
H.K. Lachowicz, A. Milewski ${ }^{+}$and $\mathrm{J}$. Wenda ${ }^{++}$ \\ Institute of Physics, Polish Academy of Sciences, al. Lotnikow 32/46, \\ 02-668 Warsacura, Poland \\ +Institute of EZectron Technology, Warsow Technical University, \\ uz. Koszykowa 75, 00-662 Warszowa, Poland \\ Solid State Physics Dept., Academy of Mining and Metalzurgy, \\ al. Mickiewicza 30, 30-059 Krakow, Poland
}

Résumé - Des possibilités d'application, autres que pour 1'enregistrement, des couches amorphes d'alliages terres rarés-métaux de transition et métal de transition-métalloide sont présentées. On considère en particulier les dispositifs générateurs d'ondes acoustiques de surface dans lesquels les films amorphes peuvent servir à l'accord des paramètres du dispositif. Une utilisation possible des films comme senseurs thermiques est aussi présentée.

Abstract - Possible non-storage applications of amorphous films of the rare earth-transition metal and transition metal-metalloid alloys are presented. Particular attention is given to the surface acoustic wave devices in which amorphous films can serve to tune the device parameters. A possible utilization of these films as thermal sensors is also presented.

\section{INTRODUCTION}

Research on amorphous magnetic films has been triggered by the paper of Chaudhari et.al. [1], published in 1973, showing that sputtered films of the Gd-Co alloys can exhibit perpendicular anisotropy and that magnetic bubbles with submicron diameters can be created in these films.

Since this discovery, intense research on amorphous films of rare earth-3d transition metal (RE-TM) alloys has been discernible. However, this activity was mainly oriented towards applications of the RE-TM films as storage media for thermomagnetic beam addressable menories, since some technological limitations made it rather questionable to utilize these films in bubble memories.

Though the main effort has been concentrated on the above-mentioned applications, there are, nevertheless, other possible ways of utilizing the amorphous RE-TM films, e.g. in the surface acoustic wave (SAW) devices, as Hall sensors, etc.

The rapid development of metallic glasses, which took place during the last decade has, despite being concentrated on ribbons, also induced an interest in amorphous M-M films (where M-metalloid). At the beginning, research on these films was concentrated on their fundamental physical properties, but shortly afterwards it has been shown that the TM-M amorphous films can also be utilized in devices, e.g. as thermal sensors, magnetic mirrors in ring-laser gyroscopes, etc.

A survey of the possible non-storage applications of both the RE-TM and TM-M amorphous films will be given in this paper. Attention will be focused on the utilization of these films in the SAW-devices. However, other possible applications will also be briefly indicated, in particular, those which have been developed by the authors' colleagues from collaborating institutions.

\section{MAGNETICALLY TUNED SAW-DEVICES}

SAW-devices are at present widely used for signal processing in the frequency band of $30 \mathrm{MHz}$ up to $1.5 \mathrm{GHz}$, giving a cheap and efficient solution in this field of application.

Deposition of a magnetostrictive film onto the surface of a piezoelectric substrate (usually $\mathrm{LiNbO}_{3}, \mathrm{LiTaO}_{3}$ or quartz, though $\mathrm{Bi}_{12} \mathrm{GeO}_{20}$ also seems to be a potential candidate for this application, owing to its good piezoelectric properties) between the interdigital transducers in a SAW-cievice (its geometry is 


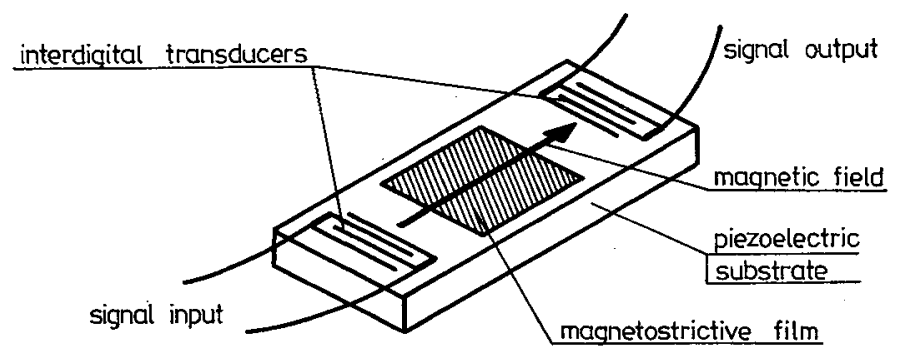

Fig. 1 - Magnetically variable SAW-delay line (after ref. [2]).

snown in Fig 1) is a technique which gives a possibility of changing the velocity of the propagating SAW by application of an external d.c.-magnetic field. These changes are due to the magnetoelastic interaction within the film and mechanical coupling between the film and the piezoelectric substrate.

The possibility of varying the SAW-velocity is desirable because of the correction of the technological scatter (after fabrication the electrical parameters of the device are fixed, since they depend solely on the geometry and spacing of the transducers, and on the electric, elastic, and piezoelectric properties of the substrate and the electrodes). Because of ageing effects (ageing rates of $(1 \div 10) \times 10^{-6}$ per year are common [2]) a possibility of periodic trimming of the device parameters to theix nominal values is also required. The possibility of tuning can also be desirable to compensate for the temperature variation of the device parameters.

On the other hand, there are applications of the SAW-devices where some degree of tuning is required, usually rapid and unpredictable.

Though tilere are well known methods of tuning the SAW-devices, for example by means of a controlled variation of the electric charge on the surface of the semiconducting layer located on the piezoelectric substrate, the use of a magnetostrictive film seems to be a very promising technique, mainly because of its technological simplicity.

\subsection{Requirements for films}

Historically, a polycrystalline Ni-film was one of the first materials evaluated for magnetic tuning of a SAW-device [3]. The changes of SAW-velocity obtained for Ni-films are in the range of $0.02 \div 0.07 \%$ but the intensity of the bias field necessary for obtaining these changes is within the $1 \mathrm{k} 0 \mathrm{e}$ range.

The dependence of the velocity variation upon the film parameters is rather complex. Theoretical approaches, simple and more rigorous, have been developed to predict the dependence of the SAW-velocity on the applied magnetic field (see e.g. [4] [5]). The common result obtained is that most of the variation of the velocity occurs in the range of field values in which a uniform rotation of the film magnetization takes place and these changes are due to the change of the elastic moduli of the magnetostrictive film (the so-called $\Delta E-e f f e c t$ ).

This result is not surprising since the velocity of bulk acoustic waves is given by $V=\left(\mathrm{C}^{\mathrm{eff}} / 0\right)^{1 / 2}$, where $\mathrm{p}$ is the density of the material in which an acoustic wave is propagating and $C^{e f f}$ is the effective elastic stiffness constant, dependent on the acoustic wave mode and the structural symmetry of the material.

An analysis of the magnetoelastic wave excitation in an amorphous film, performed by Fujii et al. [6] on the basis of a simple one-dimensional ' string model, has shown that the maximum change of the sound velocity is obtained when the direction of magnetization forms an angle of $45^{\circ}$ with the direction of sound propagation. To achieve this effect, changes of the film magnetization should occur as a result of the rotation. This could be the case if one creates in the film an anisotropy (possibly a low one) with the in-plane easy axis perpendicular to the external field direction. Such anisotropy can be induced by means of a thermomagnetic treatment. However, care should be taken when using this procedure since, according to the authors' experience, the induced anisotropy can have a rotational character (see e.g. [7]) with the time constant in the range of $5 \div 100 \mathrm{sec}$.

A relation, linking the $\Delta E$-effect with the magnetic and elastic parameters of the material, was given by Kersten [8] half a century ağo 


$$
\frac{\Delta E}{E}=\frac{E_{S}-E}{E}=\frac{9}{20 \pi} \frac{\mu \lambda_{s}^{2} E}{M_{S}^{2}},
$$

where $E_{S}$ and $E$ are the Young's modulus at the saturated and at the given field, respectively, $\mu$ - magnetic permeability, $\lambda_{S}$ - saturation magnetostriction, $M_{S}$ - saturation magnetization.

Although not universally applicable, eq. (1) gives a reasonable approximation of the experimental results. Fukamichi et al. [9] have shown that in the case of unannealed samples of the $\mathrm{Fe}_{33} \mathrm{P}_{17}$ and $\mathrm{Fe}_{86} \mathrm{~B}_{14}$ metallic glasses, the observed and calculated (using eq. (1)) values of the $\Delta E$-effect are in reasonable agreement, as can be seen in Table 1. For selection of the type of film to be used in a SAW-device,

Taole 1 - Calculated and measured values of $\Delta E / E_{D}$ (after ref. [9])

\begin{tabular}{|c|c|c|c|c|c|}
\hline Al10y & $\begin{array}{c}\lambda_{\mathrm{S}} \\
(\times 10-6)\end{array}$ & $\begin{array}{c}\mathrm{E}_{\mathrm{S}} \\
\left(\times 10^{12} \mathrm{dyn} / \mathrm{cm}^{2}\right)\end{array}$ & $\mu_{0}$ & $\left(\frac{\Delta \mathrm{E}}{\mathrm{E}_{\mathrm{D}}} \mathrm{calc}\right.$ & $\left(\frac{\Delta \mathrm{E}}{\mathrm{E}_{\mathrm{D}}}\right)$ meas. \\
\hline $\mathrm{Fe}_{83} \mathrm{P} 17$ & 30 & 1.35 & 670 & 0.09 & 0.11 \\
\hline $\mathrm{Fe}_{86}{ }^{\mathrm{B}} 14$ & 31 & 1.78 & 3500 & 0.40 & 0.43 \\
\hline
\end{tabular}

where $E_{D}$ is the Young's modulus at zero field (demagnetizing state).

the material with the most favourable parameters can be found by using eq. (1), looking for the material exhibiting the largest $\Delta E$-effect possible. As can easily be noticed by considering tnis equation, films with large magnetostriction and low magnetization would be the best choice if we want to fulfil the requirements for the maximum possible $\Delta E-$ effect.

The $\mathrm{Tb}-\mathrm{Fe}$ amorphous alloys show relatively large magnetostriction $\left(\lambda_{\mathbf{s}}=310 \times 10^{-6}\right.$ for amorphous $\mathrm{TbFe}_{2}[10]$, while for a cxystalline compound of the same composition this value is almost six times larger). Since these alloys behave like ferrimagnets (their magnetic structure is of the sperimagnetic type), conditions for a low magnetization can easily be achieved by a proper selection of the film composition (see Fig.7). Though Yamaguchi et al. [11] have shown that the use of the $\mathrm{TbFe}_{2}$ amorphous film in the SAW-device makes it possible to achieve the largest ever reported change of the SAW-velocity, $\Delta V / V=-0.27 \%$, this variation is obtainable for relatively high intensities of the magnetic field (of the order of $4 \mathrm{kOe}$ ) because of the giant anisotropy, of single-ion origin, present in these films. Application of a field of so high an intensity is unacceptable from the standpoint of construction of tuned SAW-devices, because of the over-sized magnetic bias field circuitry which snould be designed for the minimum power consumption and fast switching.

Therefore, the effort has concentrated on searching for the materials which are much softer magnetically.

It is well known that ferromagnetic metallic glasses of the Fe-based alloys show, if properly annealed, an extraordinarily low anisotropy. Typical values of magnetostriction for these alloys are of the order of $30 \times 10^{-6}$ (see e.g. Table 1). Use of the evaporated Fe-B amorphous films in the SAll-device has shown that the tuning effect is obtainable within 10 oe of the bias field, but that its maximum value does not exceed $0.03 \%$ [2]. Though $\Delta V / V$ is an order of magnitude lower than in the case of the $\mathrm{Tb}-\mathrm{Fe}$ film, this value is nevertheless adequate for many applications. It therefore seems that amorphous films of the $\mathrm{Fe}$-based alloys are the best candidates for the materials to be utilized for magnetic tuning of the SAW-devices.

Both thermal evaporation and sputtering techniques can be applied to produce a magnetostrictive layer on the surface of the piezoelectric substrate of the SAW-device. However, application of sputtered films gives better results since this technique introduces lower intrinsic stresses. The stress gives rise to effective anisotropy via the magnetoelastic interactions, which results in the easy axis of magnetization being in the film plane or perpendicular to it, depending on the sign of both the stress and the magnetostriction. Some good evidence for the presence of this effect is shown by the results of annealing of a $\mathrm{Fe}-\mathrm{B}$ amorphous film sputtered 
onto a LilvbO 3 -substrate, shown in Fig. 2 in the form of the SAW-velocity dependence on the bias field for different temperatures of the annealing performed in an argon atmosphere (using the measuring configuration shown in Fig.3). Note that the individual curves follow the shape of the $\triangle E / E$ dependence on the magnetic field (see e.g. [12]). As can be seen in Fig.2, the field at which the velocity change achieves its maximum value shifts with an increase of the annealing temperature to lower intensities, as can be expected, whereas the maximum value of $\Delta V / V$ is rather weakly dependent on tỉis temperature.

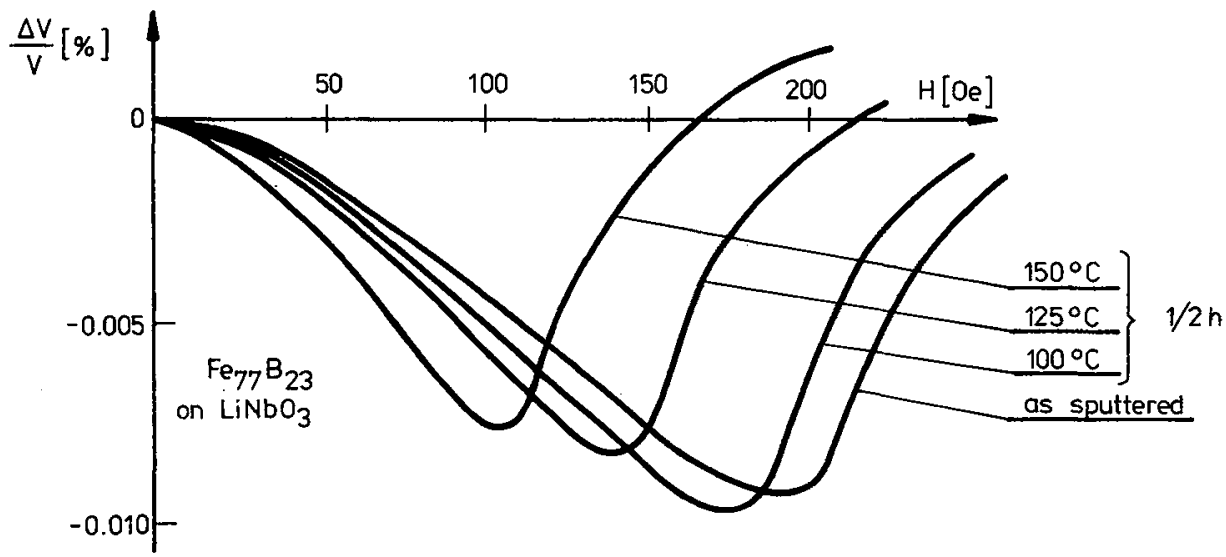

Fig. 2 - SAW-velocity change vs. applied magnetic field for different annealing temperature for a $0.7 \mu \mathrm{m}$ amorphous $\mathrm{Fe}-\mathrm{B}$ film on $\mathrm{YZ}-\mathrm{LiNbO} 3$. The frequency is $69 \mathrm{MHz}$.

An increase of the film thickness gives a greater velocity variation since a larger fraction of tine acoustic energy is carried within the film. A disadvantage of such a procedure is an increase of attenuation and dispersion. Therefore, the optimum film thickness is adjusted by a compromise and is usually of the orcier of $1 \mu \mathrm{m}$.

To get the best performances the film parameters have to be known, in particular, those appearing in eq. (1). Since Young's modulus does not change significantly with the film composition for the Fe-based alloys, the important parameters are $i_{S}, \mu$ and $\lambda_{S}$.

However, care should be taken when the magnetostriction of the film has to be measured. As a general rule for this purpose one uses the experimental methods which make it possible to determine just one magnetostriction constant, namely $\lambda_{s}$. This parameter properly describes the magnetostrictive properties of the film only if the medium is isotropic. As has been shown earlier, the amorphous films to be utilized in the SAiN-devices have to exhibit an anisotropy in the film plane. By considering an anisotropic amorphous film, Szymczak [13] has shown that the magnetoelastic tensor $B_{i j k l}$ for such a material has the form (written in the Voigt notation) determined by $\infty / \mathrm{mm}$ point symmetry

$$
[\mathrm{B}]=\left[\begin{array}{llllll}
\mathrm{B}_{11} & \mathrm{~B}_{12} & \mathrm{~B}_{13} & 0 & 0 & 0 \\
\mathrm{~B}_{12} & \mathrm{~B}_{11} & \mathrm{~B}_{13} & 0 & 0 & 0 \\
\mathrm{~B}_{31}^{1} & \mathrm{~B}_{31} & \mathrm{~B}_{33} & 0 & 0 & 0 \\
0 & 0 & 0 & \mathrm{~B}_{44} & 0 & 0 \\
0 & 0 & 0 & 0 & \mathrm{~B}_{44} & 0 \\
0 & 0 & 0 & 0 & 0 & \mathrm{~B}_{66}
\end{array}\right] \text {, }
$$

where $\mathrm{B}_{3 j}=-\mathrm{B}_{11}-\mathrm{B}_{12}, \mathrm{~B}_{66}=1 / 2\left(\mathrm{~B}_{11}-\mathrm{B}_{12}\right), \mathrm{B}_{33}=-2 \mathrm{~B}_{13}$,

In this case, the magnetoelastic interactions are determined by four independent parameters instead of the one sufficient for an isotropic medium. The first indirect indication that linear magnetostriction could have an anisotropic character was given by Twarowski et al. [14] for the case of amorphous $\mathbf{r}$.f.-sputtered Gd-Co films. Experimental evidence for this behaviour has been given by zuberek et al. [15], who investigated the $\mathrm{Fe}_{1-\mathrm{X}_{\mathrm{X}} \mathrm{X}}$ amorphous films by means of the strain modulated ferro- 
magnetic resonance (SMFMR) method, which allows one to calculate more than one magnetostriction constant. The results obtained in this experiment (shown in Table 2) indicate a considerable departure of the magnetostriction from isotropic behavior, since for isotropic magnetostriction the following relation should be fulfilled: $\mathrm{B}_{11} / \mathrm{B}_{12}=-2$.

Table 2 - Measured values of $\mathrm{B}_{11} / \mathrm{B}_{12}$

for $\mathrm{Fe}_{1-\mathrm{X}} \mathrm{B}_{\mathrm{X}}$ amorphous films (after ref.[15]).

\begin{tabular}{|c|c|c|c|c|c|}
\hline$x$ & 0.47 & 0.50 & 0.50 & 0.55 & 0.60 \\
\hline $\mathrm{B}_{11} / \mathrm{B}_{12}$ & -2.96 & -2.23 & -2.09 & -2.82 & -2.57 \\
\hline
\end{tabular}

The SMFMR method is rather inconvenient for standard measurements. Though other methods allow one to determine only one magnetostriction constant $-\lambda_{S}$, they should nevertheless be used to estimate the magnetostrictive behaviour of the films of interest.

Among the known methods, the cantilever-capacitance one, developed by Klokholm [16], seems to be the most suitable (see e.g. [17]). However, it has recently been shown that the so-called small-angle magnetization rotation method [18] can be used successfully if the film is deposited onto a polyester substrate.

To determine the change of sound velocity in a magnetically tuned SAW-device one should use the circuit shown in Fig.3. This change is calculated as a function of

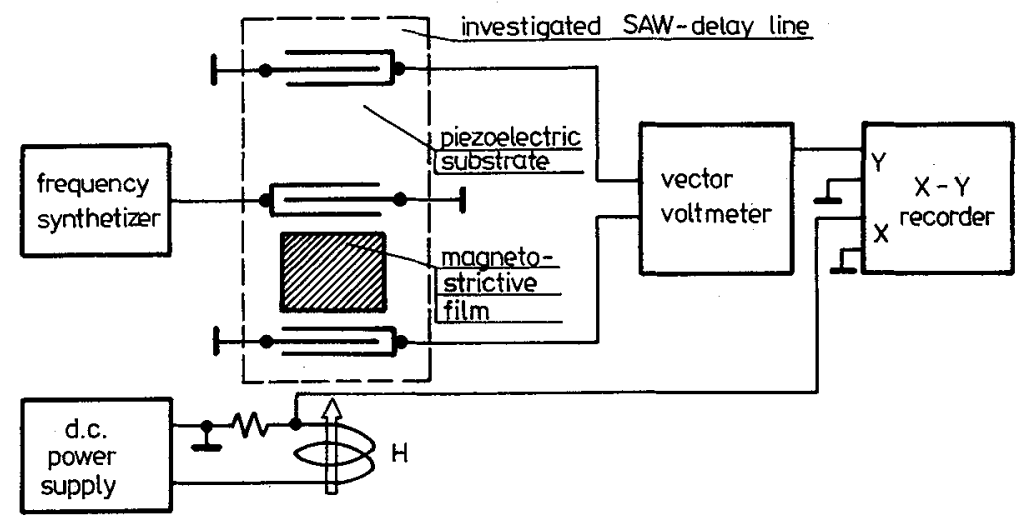

Fig. 3 - Measuring configuration of SAW-velocity change.

the external magnetic field from the measured phase difference between the signals obtained at the outputs of two symmetric SAW-delay 1ines, one of them with the deposited film, both placed on the same piezoelectric substrate. As a result of this design, the influence of the temperature is cancelled. The details of the measuring set-up are described elsewhere [19].

\subsection{An application of magnetically tuned SAW-devices}

As has been said earlier, tuning of the SAW-devices is desired in order to correct the technological inaccuracies, the changes of parameters resulting from the ageing effects and to control the temperature variation.

The first two of these requirements can easily be fulfilled by applying a permanent magnet of sufficient intensity, which will influence the sound velocity via the deposited film. A proper placing of this magnet gives the required amount of tuning. Control of the temperature variation of the SAW-device parameters can be realized by using an electromagnet in conjunction with a thermocouple sensor. This solution seems to be a good alternative to keeping the SAW-device in a thermostat.

Magnetically tuned SAW-devices can be used in systems in which a small, but rapid and unpredictable amount of tuning is necessary. A good example of these applications is the adaptive antenna array where the direction of the beam is guided by means of magnetically tuned SAW-delay lines [2]. An attractive alternative to conventional approaches seems to be a magnetically tuned SAW-oscillator capable of tracking a high speed Doppler target [2].

Since the SAW-device can act as a filter with a very narrow frequency band, if only the pattern of the interdigital transducer. is properly designed, the device can 
be utilized in an oscillating circuit, shown in Fig, 4. A laboratory design of a $69 \mathrm{MHz}$ oscillator based on this idea has shown a behaviour which seems promising from the point of view of the possible utilization of these devices in frequency synthetizers. The generated signa1, as displayed on a wobbulator screen, is shown in Fig. 5. Lse of the narmonics of the fundamental frequency, possible in the case of
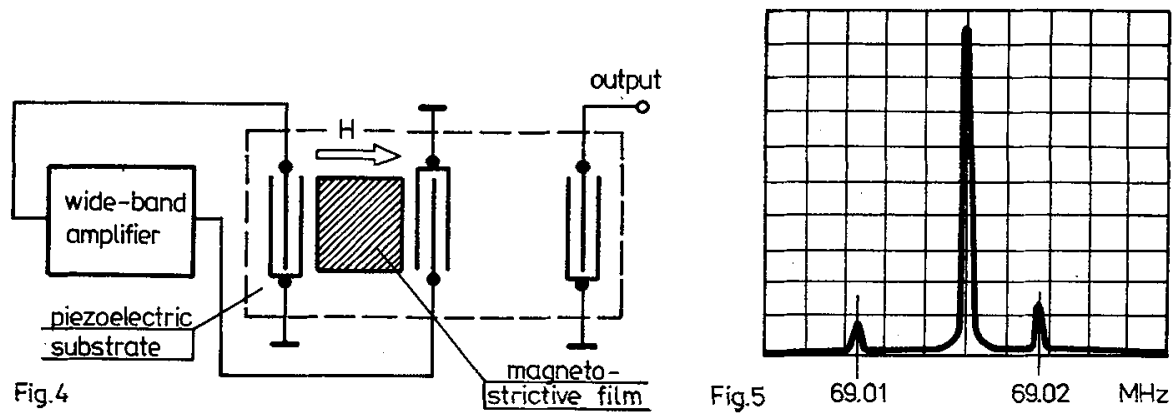

Fig. 4 - SAW-oscillator circuit.

Fig. 5 - Generated signal in cixcuit designed as in Fig. 4.

a special design of interdigital transducer patterns, makes it possible, according to the authors' estimate, to generate frequencies of up to $1.5 \mathrm{GHz}$. However, fabrication of the pattern requires a very high accuracy (of the order of $0.1 \mu \mathrm{m}$ ), which can only be achieved by means of electron or x-ray Iithography. Application of an amorphous film in this device allows one to tune the frequency and/or to compensate for the temperature variation of the frequency.

\section{THERMAL SENSORS}

A simple experiment performed [20] on the domain structure of the RE-TM amorphous films has shown that in a ferrimagnetic film with perpendicular anisotropy, a sudden reversal of magnetization, whici could be expected when the temperature passes through the compensation point, occurs only if the external applied field $\mathrm{H}_{\mathrm{a}}$ is of sufficient intensity. This intensity is given by $\left|M_{R E}{ }^{-M_{T M}}\right| \cdot H_{a}>E_{a}$, where $M_{R E}$ and $M_{T M}$ are the magnetizations of the RE, and TM-subnetworks, respectively, $E_{\mathrm{a}}$-the energy, directly related to the perpendicular anisotropy energy.

This phenomenon manifests itself clearly if one studies the temperature dependence of the Hall voltage. It is well known that this voltage changes its sign when the temperature of the film passes through the compensation point (see e.g. [20]). Since this effect is usualiy observed under conditions of a high applied field, the thermal hysteresis which can be predicted by analyzing the inequality given above has not been noticed. More detailed investigations performed on the Ho-Co amorphous films [21] have shown the existence of thermal hysteresis of the Hall voltage. (Fig.6). The width of the hysteresis loops is field-dependent, as could be expected

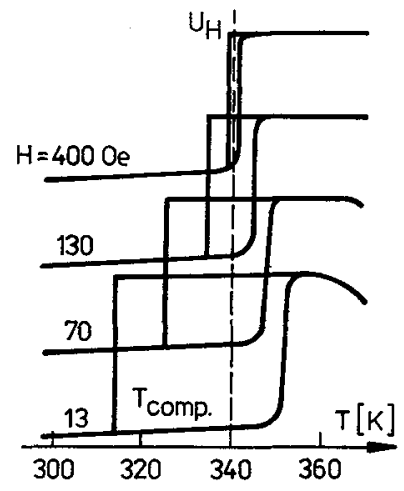

Fig. 6 - Thermal hysteresis loops of the Hall voltage (after ref. [21]). 
when one considers the inequality discussed above and the known temperature dependence of the net-magnetization of the ferrimagnetic film (Fig. 7).

The effect described above can be utilized

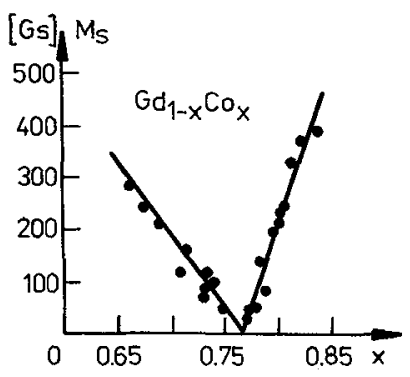

Fig. 7 - Saturation magnetization vs. Co-concentration for $\mathrm{Gd}_{1-\mathrm{x}} \mathrm{Co}_{\mathrm{X}}$ amoxphous films (room temperature). in sensors as a safety catch for switching off a device which may be damaged on overheating and switching it on after it has cooled down to a safe temperature, as has been suggested in [21]. The temperature range required can easily be adjusted by selecting the film composition and/or the magnetizing field.

It has been shown that the $\mathrm{Fe}-\mathrm{B}$ and Fe-P amorphous alloys exhibit Invar behaviour in a wide temperature range below the Curie temperature [9]. Invar materials have extraordinarily small thermal expansion coefficients; e.g. $\alpha=2.3 \times 10^{-6}$ $\left(0 \div 40^{\circ} \mathrm{C}\right)$ in the case of $\mathrm{Fe}_{85} \mathrm{~B}_{15}[9]$, whereas for crystalline $\mathrm{Fe}$, for example, $\alpha=11.7 \times 10^{-6}\left(20^{\circ} \mathrm{C}\right)$. This behaviour, along with their excellent mechanical properties, provides a unique opportunity to utilize amorphous films in bimetallic structures. In addition, use of sputtering or evaporation techniques can easily solve the problem of combining amorphous and crystalline layers. Since sensitivity of bimetallic sensors is proportional to the difference of thermal expansion coefficients of the component used, one can expect a significant improvement of this parameter when utilizing amorphous films instead of conventional materials.

The idea described above has recently been realized (Stobiecki and Stobiecki, to be pubished) in the form of a bimetallic structure composed of a Fe-B amorphous film sputtered onto a crystalline Al-foil $\left(\alpha=25 \times 10^{-6} ;\right.$. The very high sensitivity of this structure to small temperature changes has been confirmed.

This result allows us to expect that bimetallic structures utilizing amorphous TM-M films will find their way into various precise control instruments.

\section{OTHER POSSIBILITIES OF APPLICATION}

Amorphous films of both types, RE-TM and TM-M, can be utilized as magnetic mirrors in ring-1aser gyroscopes [22], giving protection against the undesirable effect which manifests itself as the lock-in of the two light beams to a common frequency. Since amorphous films exhibit an extraordinarily large spontaneous hall effect they can be applied as sensitive Hall detectors.

More sophisticated use of these films has also been reported, e.g. the propagation of bubbles created in the films, using the so-called "domain-drag effect $[23]$.

\section{ACKNOWLEDGMENT}

The authors wish to gratefully acknowledge the support given for this work by the Government Grant PR-3.19.

Thanks are due to a number of colleagues from the authors' Institutes as well as. from collaborating institutions, in particular, to Dr. H.Jankowski, Dr. F.Stobiecki, Dr. A.Kułak, Mr. J.Sokulski, and Mr. J.Samula, for their cooperation in this work.

A word of special thanks is given to Prof. H.Szymczak for a critical reading of the manuscript.

\section{References}

[1] P.Chaudhari, J.J.Cuomo, R.J.Gambino, IBiv J.Kes.Dev. 11,66 (1973) .

[2] D.C.Webb, D.W.Forester, A.K.Ganguly, C.Vittoria, IEEE Trans.lagn. MAG-15, $1410(1979)$.

[3] A.K.Ganguly, K.L.Davis, D.C.Webb, C.Vittoria, Electron.Letters, 11,610 (1975).

[4] D.C.Webb, D.W.Forester, A.K.Ganguly, C.Vittoria, J.Appl.Phys. 47,2696 (1976).

[5] M.Levy, H.Yashida, J.Magn.Mag.Mat. 35,139 (1983). 
[6] T.Fujii, T.Kamura, T.Miyama, M.Inoue, Jap.J.App.Phys. 22,79 (1983) .

[7] R.F.Soohoo, Magnetic Thin Films, Harper and Row, New York, 1965, p.125.

[8] M.Kersten, Z.Phys. 85, 708 (1933).

[9] K.Fukamichi, T.Masumoto, M.Kikuchi, IEEE Trans.Magn. MAG-15, 1404 (1979).

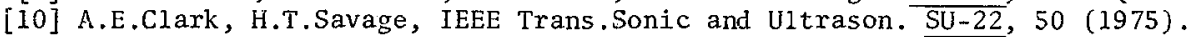

[11] M.Yamaguchi, K.Y.Hashimoto, H.Kogo, M.Naoe, IEEE Trans.Magn. MAG-16, 916 (1980).

[12] B.S.Berry, W.C.Pritchet, Phys.Rev.Letters, 34, 1022 (1975).

[13] H.Szymczak, J.Magn.Magn.Mat. 15-18, 585 (19 $\overline{80}$ ).

[14] K.Twarowski, H.K.Lachowicz, M.Gutowski, H.Szymczak, Phys.Stat.So1. (a), 63, 103 (1981).

[15] R.Żuberek, F.Stobiecki, J.Wosik, Phys.Stat.Sol. (a), 82, K177 (1984).

[16] E.Klokholm, IEEE Trans.Magn. MAG-12, 819 (1976).

[17] H.K.Lachowicz, H.Szymczak, J.Magn.Mag.Mat. 4l, 327 (1984).

[18] K.Narita, J.Yamasaki, H.Fukunage, IEEE Trans.Magn. MAG-16, 435 (1980).

[19] J.Samuła, Elektronika, nr 9, 32 (1984), in Polish.

[20] H.K.Lachowicz, IEEE Trans .Magn. MAG-20, 1417 (1984).

[21] H.Ratajczak, I.Gościańska, Phys. .Stat.Sol. (a), 73, 633 (1982).

[22] J.J.Krebs, W.G.Maisch, G.A.Prinz, D.W.Forester, IEEE Trans. on Magn. MAG-16, $1179(1980)$.

[23] J.C.Deluca, R.J.Gambino, A.P.Malozemoff, IEEE Trans.on Magn. MAG-14, 500 (1978) . 